\title{
Зарождение трехмерных островков Ge на структурированной поверхности $\mathrm{Si}(100)$
}

\author{
(C) С.А. Рудин ${ }^{1}$, Ж.В. Смагина ${ }^{1}$, В.А. Зиновьев ${ }^{1}$, П.Л. Новиков ${ }^{1,2}$, А.В. Ненашев ${ }^{1,2}$, \\ E.E. Родякина ${ }^{1,2}$, А.В. Двуреченский ${ }^{1,2}$ \\ ${ }^{1}$ Институт фризики полупроводников им. А.В. Ржанова Сибирского отделения Российский академии наук, \\ 630090 Новосибирск, Россия \\ ${ }^{2}$ Новосибирский государственный университет, \\ 630090 Новосибирск, Россия \\ E-mail: rudin@isp.nsc.ru
}

(Получена 25 апреля 2018 г. Принята к печати 7 мая 2018 г.)

Исследовано зарождение трехмерных островков $\mathrm{Ge}$, формируемых на структурированной поверхности $\mathrm{Si}$ в виде массива ямок округлой формы. Обнаружено, что зарождение островков Ge происходит внутри ямок с остроконечным дном и по периметру ямок с плоским дном. Данный эффект обусловлен различием в распределении упругой деформации на границе $\mathrm{Ge} / \mathrm{Si}$ в зависимости от формы дна ямок. Результаты моделирования роста показали, что для ямок с острым дном наиболее релаксированная область находится по центру дна ямки, где и происходит зарождение островков. Тогда как для ямок с плоским дном наиболее релаксированные области смещаются со дна ямок к их краям, что приводит к зарождению островков по их периметру.

DOI: 10.21883/FTP.2018.11.46596.18

\section{1. Введение}

Гетероструктуры с квантовыми точками (КТ) вызывают повышенный интерес во всем мире в связи с их уникальными физическими свойствами и возможностью создания на их основе светоизлучательных приборов и фотоприемников [1-3]. Прогресс, наблюдаемый в создании наноструктур пониженной размерности, связан с использованием эффектов самоорганизации. Эффект спонтанного образования трехмерных островков при гетероэпитаксии материалов с большим рассогласованием постоянных решеток (> $2 \%$ ) позволяет получать массивы квантовых точек с высокой плотностью $[3,4]$. В связи с тем что зарождение наноостровков $\mathrm{Ge}$ на $\mathrm{Si}$ происходит хаотично, дисперсия (разброс) по размерам наноостровков в таких структурах достигает 20\%, а их расположение в плоскости роста не поддается точному контролю [5-10]. Для решения этой проблемы было предложено использовать поверхность с предварительно созданными местами для зарождения наноостровков (затравочными областями) в виде массива ямок, периодически расположенных на поверхности подложки [11-14]. Ямки служат стоком для осажденных атомов, что приводит к зарождению наноостровков в их окрестности. При осаждении Ge на подобных структурированных подложках $\mathrm{Si}$ наблюдается упорядочение островков в плоскости роста и уменьшение разброса наноостровков по размерам по сравнению с гетероэпитаксией на гладкой поверхности [15].

Рост Ge/Si-структур на поверхности с предварительно созданными местами для зарождения трехмерных островков включает в себя сложные механизмы, не принимаемые во внимание в упрощенной модели роста по механизму Странского-Крастанова. Эти механизмы вклю- чают в себя зависимость релаксации механических напряжений от геометрической формы ямок, поверхностную анизотропию, различные реконструкции на стенках ямок. В работе Vastola и др. исследовалась зависимость пространственного расположения трехмерных островков Ge от угла наклона боковых стенок ямок, предварительно сформированных на поверхности $\mathrm{Si}(100)$ [16]. Было показано, что зарождение островков происходит внутри ямок только при малых углах наклона их стенок $\left(<32^{\circ}\right)$, в то время как для больших углов наклона боковых стенок островки растут снаружи ямок. В этой работе был проведен детальный расчет распределения упругой энергии для различных морфологий $\mathrm{Ge}-с л о я ~$ в ямках. Полученные результаты позволили авторам работы сделать вывод, что релаксация упругих напряжений является основным фактором, определяющим место зарождения трехмерных островков. Формирование более чем одного островка на каждую ямку наблюдалось несколькими исследовательскими группами [16-20]. Были предложены различные механизмы в зависимости от методов создания структурированной поверхности и условий роста. Zhong и другие наблюдали формирование одиночных островков на дне ямок, за которым следовало формирование симметричной группы Ge-островков по их периметру [18]. Механизм формирования островков вокруг ямок в большей степени объяснялся геометрией поверхности, чем упругими эффектами [18]. Авторы работы [17] наблюдали формирование групп из четырех Ge hut-кластеров в внутри каждой ямки. Для объяснения данного результата авторы предположили, что изломы на боковых стенках являются предпочтительными местами для собирания адатомов Ge. Морфологическая эволюция как ямок, так и $\mathrm{SiGe-островков} \mathrm{была}$ исследована авторами работы [21]. На стадии роста 
буферного слоя $\mathrm{Si}$ боковые стенки ямок представляли собой грани $\{113\}$, в то время как их углы огранялись плоскостями $\{103\}$. В дополнение указывалось наличие более мелких граней $\{11 n\}$. С дальнейшим увеличением количества осаждаемого $\mathrm{Si}$ форма ямок изменялась в сторону перевернутых усеченных пирамид. Островки, формируемые во время осаждения различного количества $\mathrm{Ge}$, изменяли форму от перевернутых пирамид к усеченным, содержащим грани (001) и $\{105\}$, и в итоге к пирамидам, ограненным плоскостями $\{105\}$. Для рассмотренных случаев островки наблюдались только по центру ямок, в то время как между ямками островки не зарождались. Значительные изменения в морфологии поверхности, такие как поворот ямки в процессе осаждения $\mathrm{Ge}$, наблюдались с помощью численного моделирования в работе Salvalaglio и др. [22]. При моделировании процесса гетероэпитаксии учитывалась зависимость поверхностной энергии от направления. Было обнаружено, что упругие эффекты могут приводить к образованию дополнительных граней на боковой поверхности ямок, влияющих на зарождение островков.

В данной работе изучено влияние формы ямок на пространственное расположение трехмерных наноостровков $\mathrm{Ge}$. Моделирование методом Монте-Карло воспроизводит экспериментально наблюдаемую зависимость мест преимущественного зарождения трехмерных островков $\mathrm{Ge}$ от формы дна ямок. Было установлено, что изменение формы ямки во время роста смачивающего слоя $\mathrm{Ge}$ оказывает существенное влияние на место зарождения трехмерных островков Ge.

\section{2. Методика эксперимента}

Структурированная поверхность $\mathrm{Si}(100)$ с затравочными областями в виде ямок, упорядоченных в двумерную квадратную решетку с периодом 2 мкм, создавалась с помощью электронно-лучевой литографии (ЭЛЛ) и плазмохимического травления (ПХТ). Для этого остросфокусированным электронным пучком на предварительно нанесенном резисте толщиной 200 нм на поверхности $\mathrm{Si}(100)$ создавался рисунок в виде локальных экспонированных областей. После проявления резист служил маской при переносе рисунка на подложку $\mathrm{Si}(001)$ в процессе ее ПХТ. Травление проводилось в плазме $\mathrm{SF}_{6}+\mathrm{CFCl}_{3}$ в течение 25-45 с. Таким образом, была получена структурированная поверхность $\mathrm{Si}$, содержащая разреженный массив ямок, имеющих круглую форму. Диаметр ямок составлял 200 нм, глубина ямок определялась временем проведения ПХТ и лежала в диапазоне 20-160 нм.

Осаждение $\mathrm{Ge}$ на структурированную подложку $\mathrm{Si}(100)$ проводилось в установке молекулярно-лучевой эпитаксии (МЛЭ) SIVA-21 фирмы Riber. Перед ростом структур подложка проходила двойную очистку поверхности: химическую обработку и покрытие защитным окислом, а далее удаление защитного слоя $\mathrm{SiO}_{2}$ в установке МЛЭ при температуре $750^{\circ} \mathrm{C}$ в слабом потоке $\mathrm{Si}$ [23]. Процесс удаления окисла контролировался с помощью дифракции быстрых электронов. После 8-10 мин появлялись выраженные рефлексы $(2 \times 1)$, указывающие на то, что поверхность становилась атомарно чистой. Затем осаждался буферный слой $\mathrm{Si}$ толщиной 40 нм при постепенном повышении температуры роста от 250 до $500^{\circ} \mathrm{C}$ с последующим отжигом при $700^{\circ} \mathrm{C}$ в течение 10 мин. На структурированную поверхность с выращенным буферным слоем $\mathrm{Si}$ осаждался слой $\mathrm{Ge}$ толщиной около 4 монослоев (MC) со скоростью $0.005 \mathrm{Hм} / \mathrm{c}$ при температуре $700^{\circ} \mathrm{C}$, что обеспечивало зарождение трехмерных Ge-наноостровков внутри или по периметру ямок.

Морфология структурированных $\mathrm{Si}(100)$-подложек и выращенных однослойных структур с упорядоченными $\mathrm{GeSi}$ KT была исследована с помощью атомно-силового микроскопа (ACM) Solver PRO фирмы NT-MDT и сканирующего электронного микроскопа (СЭМ) LEO 1430 фирмы LEO Electron Microscopy LTD.

\section{3. Описание модели}

Для выявления механизмов зарождения трехмерных островков $\mathrm{Ge}$ на структурированной поверхности $\mathrm{Si}$ было проведено моделирование процесса роста методом Монте-Карло (МК). Использовалась разработанная ранее модель гетероэпитаксии [24]. В основе модели лежит трехмерная алмазоподобная кристаллическая решетка. Каждый узел решетки может быть занят атомами $\mathrm{Si}$, $\mathrm{Ge}$ или быть пустым. Для учета упругой деформации каждому атому присвоена дополнительная характеристика, меняющаяся во времени, - вектор смещения относительно радиус-вектора идеальной кристаллической решетки $\mathrm{Si}$. Рост моделируется как последовательность элементарных событий осаждения и диффузионных прыжков, случайно выбираемых согласно их вероятностям. Алгоритм содержит три типа событий: добавление нового атома (осаждение), диффузионный прыжок атома по поверхности (перенос атома из одного узлового положения в другое) и случайные смещения атома вблизи его равновесного положения (новый тип событий, предложенный в модели, который позволяет избежать вычисления полной упругой энергии всей системы). Вероятность осаждения определяется требуемой скоростью роста. Вероятность диффузионного прыжка выбирается таким образом, чтобы удовлетворять двум условиям: 1) вероятность прыжка зависит только от положений ближайших соседей прыгающего атома (в пределах второй координационной сферы); 2) вероятности прямого и обратного прыжков удовлетворяют принципу детального равновесия. Энергия активации диффузионного перехода в нашей модели содержит отрицательное слагаемое, пропорциональное числу межатомных связей и числу пар вторых соседей, и положительное слагаемое, равное упругой энергии, выраженной в форме по- 

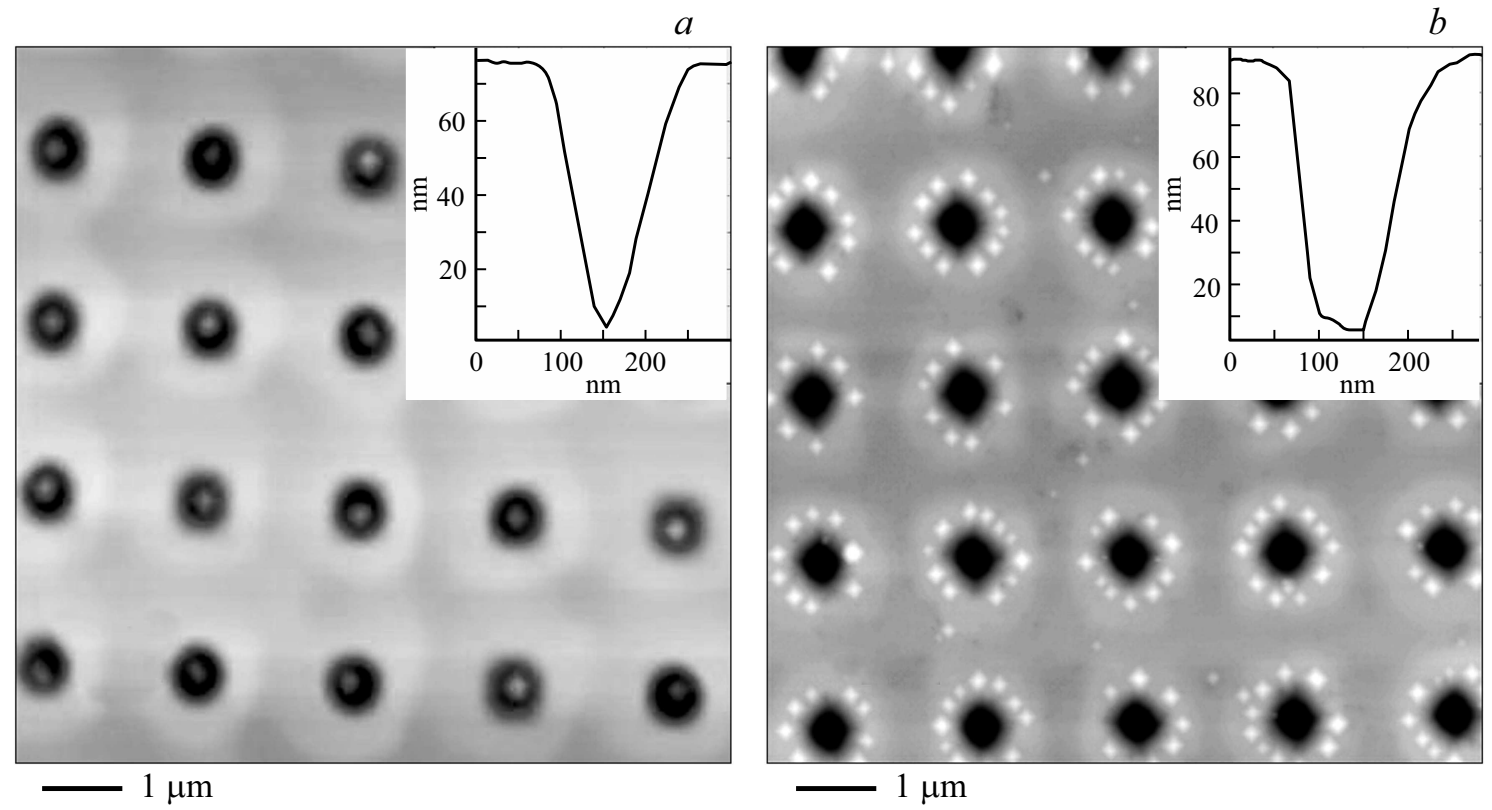

Рис. 1. СЭМ-изображения пространственно-упорядоченных трехмерных наноостровков $\mathrm{GeSi}$, полученных осаждением $\sim 4 \mathrm{MC}$ Ge при температуре $700^{\circ} \mathrm{C}$ на структурированные подложки $\mathrm{Si}(100)$ с рельефом поверхности в виде ямок округлой формы, расположенных в узлах квадратной решетки с периодом 2 мкм. Профиль ямок после плазмохимического травления в виде: $a$ - перевернутых треугольников с острой вершиной; $b-$ трапециевидная с плоским дном. На вставках - соответствующие АСМ-профили ямок.

тенциала Китинга [25]. Величины случайных смещений подчиняются распределению Больцмана. Детали модели описаны в $[24,26]$.

\section{4. Результаты и обсуждение}

С помощью атомно-силовой и сканирующей электронной микроскопии была исследована зависимость расположения мест преимущественного зарождения $\mathrm{Ge}-$ наноостровков на структурированной поверхности $\mathrm{Si}$ от формы дна ямок. Перед ростом $\mathrm{Ge} / \mathrm{Si}$-структур методом АСМ исследовались профили ямок после плазмохимического травления. Были отобраны два типа ямок одинаковой глубины: профиль которых представлял собой перевернутый треугольник с острой вершиной (рис. 1,a) и ямки с трапециевидной формой профиля с шириной нижнего основания 20-50 нм (рис. $1, b)$. После роста буферного слоя $\mathrm{Si}$ и осаждения $4 \mathrm{MC} \mathrm{Ge} \mathrm{при} \mathrm{температуре}$ $700^{\circ} \mathrm{C}$ на подложках с разным типом ямок было обнаружено, что трехмерные островки Ge расположены внутри ямок, имеющих профиль перевернутых треугольников с острой вершиной (рис. $1, a)$. При этом трехмерные островки имеют форму пирамид с прямоугольным основанием, стороны которого ориентированы в направлениях $<100>$. Размеры наноостровков для данного случая $150 \pm 5$ нм (основание) и $15 \pm 0.5$ нм (высота). В структурах с ямками с трапециевидной формой профиля происходит обрамление ямок несколькими островками (островки растут по периферии ямок, рис. $1, b$ ). В этом случае островки также имеют пирамидальную форму с латеральным размером основания $100 \pm 10 \mathrm{Hм}$, среднее количество островков, обрамляющих ямку, достигает 10.

Для объяснения экспериментально полученной зависимости положения трехмерных островков Ge от формы дна ямок, а также выявления механизмов зарождения наноостровков было проведено МК моделирование гетероэпитаксиального роста $\mathrm{Ge}$ на $\mathrm{Si}(100)$. В качестве модельной структуры использовался участок кристалла $\mathrm{Si}$ размерами $27 \times 27 \times 13.5$ нм с периодическими граничными условиями. Поверхность подложки имела ориентацию (100). В центре подложки располагалась ямка с треугольным или трапециевидным профилем. Диаметр ямок составлял 13.5 нм, глубина - 5 и 4 нм для треугольного и трапециевидного профиля соответственно. Угол наклона боковых стенок составлял $45^{\circ}$. Хотя линейные размеры модельных структур примерно на порядок меньше, чем в эксперименте, распределение механических напряжений остается адекватным в силу самоподобного поведения упругих полей.

Моделирование осаждения Ge проводилось при температуре $450^{\circ} \mathrm{C}$ со скоростью $0.1 \mathrm{MC} / \mathrm{c}$. Температура моделирования МК была выбрана более низкой, чем в эксперименте, для того чтобы обеспечить соответствующее экспериментальным условиям поведение поверхностной атомной диффузии в системе с уменьшенными геометрическими размерами ямок. Количество осажденного $\mathrm{Ge}$ составляло $4 \mathrm{MC}$.

На рис. 2 приведены расчетные распределения упругой энергии в слое $\mathrm{Ge}$, расположенного поверх ямки 
с треугольным профилем, на различных стадиях роста по количеству осажденного Ge. Цветом обозначена величина энергии, приходящейся на 1 атом Ge. Атомы Si окрашены в серый цвет. Рост островков наиболее энергетически выгоден там, где упругая энергия атомов Ge минимальна. Распределение упругой энергии меняется с увеличением количества осажденного Ge. Однако наиболее релаксированной областью в течение всего процесса осаждения остается дно ямки. После осаждения $3 \mathrm{MC} \mathrm{Ge} \mathrm{зарождается} \mathrm{трехмерный} \mathrm{островок}$ внутри ямки (рис. $2, b$ ). В случае ямок с трапециевидным профилем ситуация кардинально меняется (рис. 3). На начальных стадиях осаждения (осаждено менее 3 MC) наиболее релаксированные области расположены на границе плоского дна и стенок ямки (рис. 3,a). В процессе дальнейшего осаждения эти области смещаются вверх вдоль стенок ямки по направлению к ее внешним границам. При $3.5 \mathrm{MC}$ Ge зарождаются трехмерные островки на краях ямки (рис. 3,b). Таким образом, смещение наиболее релаксированных областей на стадии роста смачивающего слоя $\mathrm{Ge}$ определяет места зарождения трехмерных островков. Отметим, что на начальных стадиях роста Ge эти области находятся около дна ямки. Увеличение количества осажденного $\mathrm{Ge}$ приводит не только к огранке ямок, но и изменяет распределение напряжений в смачивающем слое $\mathrm{Ge}$.

Расчетная морфология поверхности, полученная при осаждении 4 MC Ge на структурированную поверхность $\mathrm{Si}$ с треугольным и трапециевидным профилем ямок, изображена на вставках к рис. $2, b$ и $3, b$ соответственно. Цветом обозначена высота рельефа поверхности. В случае ямки с треугольным профилем одиночный трехмерный островок Ge зарождается в центре ямки (см. вставку на рис. 2,b). В случае ямки с плоским дном (см. вставку на рис. $3, b$ ) моделирование роста Ge при тех же условиях приводит к формированию группы из 4 трехмерных наноостровков $\mathrm{Ge}$, расположенных вне ямки по ее периметру, в то время как по центру ямки островок не зарождается.

Результаты МК моделирования позволяют нам объяснить различие в положении трехмерных островков $\mathrm{Ge}$, наблюдаемое в эксперименте. Острое дно вносит вклад в эффективную релаксацию слоя Ge внутри ямки. В данной области зарождение трехмерных островков $\mathrm{Ge}$ энергетически более выгодно, чем снаружи. Увеличение ширины дна ямки приводит к появлению специфичного процесса, который изменяет положение мест зарождения трехмерных островков. Наиболее релаксированные области изначально располагаются на дне ямки и с увеличением количества осажденного Ge смещаются к внешней границе ямки. Данный эффект обусловлен изменением морфологии смачивающего слоя $\mathrm{Ge}$, покрывающего стенки ямки, в процессе роста. Таким образом, формирование островков Ge на границе ямок оказывается энергетически выгодным по сравнению с зарождением внутри ямок.

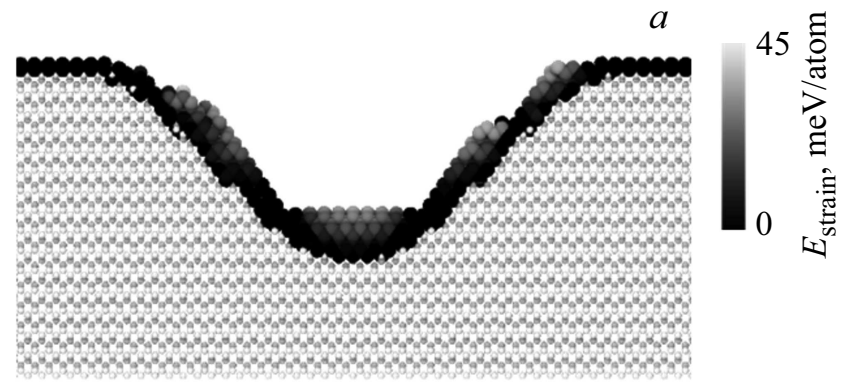

$b$

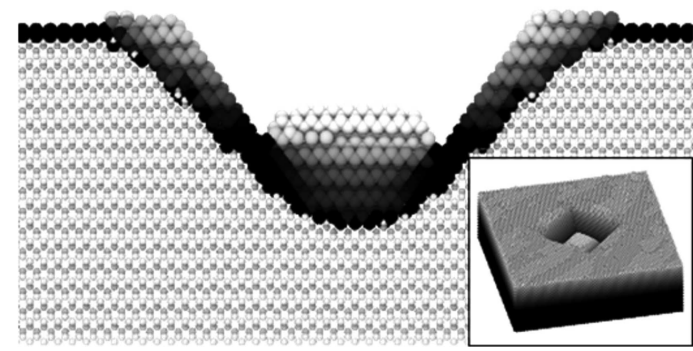

Рис. 2. Распределение упругой энергии в Ge на ямке с треугольным профилем при разном количестве осажденного материала, рассчитанное в процессе МК моделирования. Количество осажденного $\mathrm{Ge}: a-2.5 \mathrm{MC} ; b-3.5 \mathrm{MC}$. Цветом обозначено изменение упругой энергии, приходящейся на 1 атом Ge. Атомы Si окрашены в серый цвет. Моделировалось осаждение $\mathrm{Ge}$ со скоростью $0.1 \mathrm{MC} / \mathrm{c}$ при температуре $450^{\circ} \mathrm{C}$. На вставке приведено трехмерное изображение структуры после осаждения $4 \mathrm{MC} \mathrm{Ge}$.
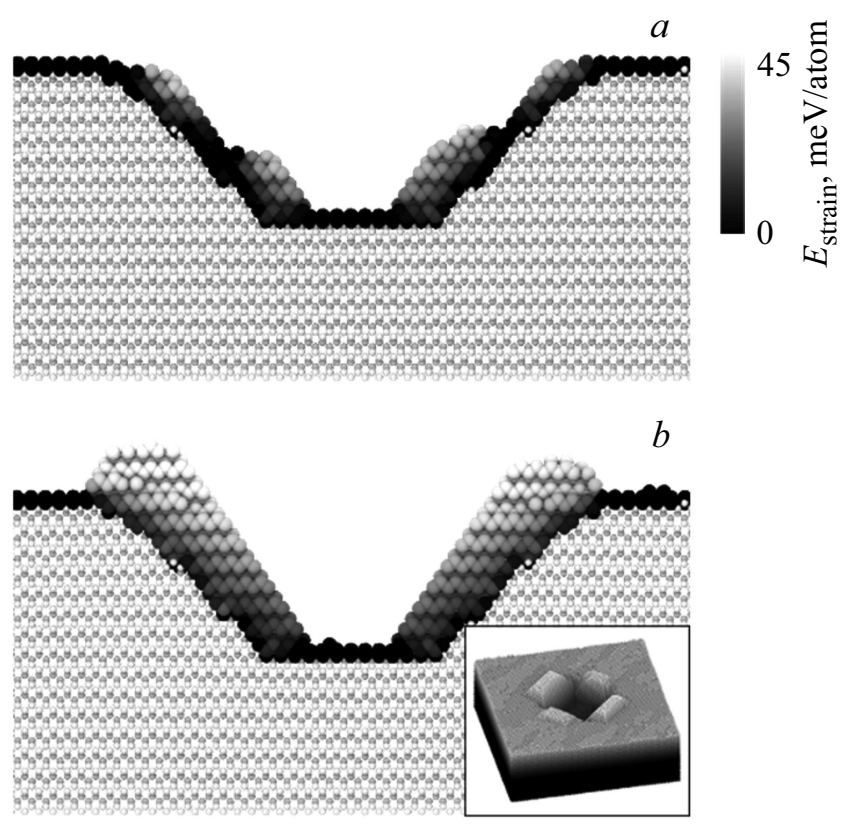

Рис. 3. Распределение упругой энергии в Ge на ямке с трапециевидным профилем при разном количестве осажденного материала, рассчитанное в процессе МК моделирования. Количество осажденного $\mathrm{Ge}: a-2.5 \mathrm{MC} ; b-3.5 \mathrm{MC}$. Цветом обозначено изменение упругой энергии, приходящейся на 1 атом Ge. Атомы Si окрашены в серый цвет. Моделировалось осаждение Ge со скоростью $0.1 \mathrm{MC} / \mathrm{c}$ при температуре $450^{\circ} \mathrm{C}$. На вставке приведено трехмерное изображение структуры после осаждения $4 \mathrm{MC} \mathrm{Ge.}$ 


\section{5. Заключение}

Установлено влияние формы дна затравочных областей (ямок) на образование наноостровков $\mathrm{Ge}$, выращиваемых на поверхности $\mathrm{Si}(100)$ с предварительно созданным массивом ямок округлой формы. Обнаружено, что островки Ge зарождаются внутри ямок, имеющих профиль перевернутых треугольников с острой вершиной. В случае, когда ямки имеют трапециевидную форму профиля с плоским дном, островки растут по периферии ямок. Различное положение островков определяется изменением распределения упругих напряжений в ямках на стадии их заполнения $\mathrm{Ge}$ в процессе двумерного роста. С помощью моделирования Монте-Карло показано, что в случае ямок с плоским дном наиболее релаксированные области смещаются со дна ямок к их краям, предшествуя стадии зарождения Ge-островков. $\mathrm{B}$ результате зарождение островков Ge происходит по периметру ямок. В случае ямок с острым дном наиболее релаксированная область находится по центру ямки с самого начала роста и до формирования $\mathrm{Ge}-$-островков.

Авторы выражают благодарность В.А. Армбристеру за рост $\mathrm{Ge} / \mathrm{Si}$-структур методом молекулярно-лучевой эпитаксии, Б.И. Фомину за проведение плазмохимического травления. Исследование выполнено за счет гранта Российского научного фонда (проект № 14-12-00931-П) в части разработки методов, роста структур и моделирования. Технология структурирования поверхности методом электронно-лучевой литографии разработана при поддержке РФФИ (грант № 16-38-00851-мол-а).

\section{Список литературы}

[1] Н.Н. Леденцов, В.М. Устинов, В.А. Щукин, П.С. Копьев, Ж.И. Алфёров, Д. Бимберг. ФТП, 32 (4), 385 (1998).

[2] A.I. Yakimov, A.V. Dvurechenskii, A.I. Nikiforov. J. Nanoelectron. Optoelectron., 1 (2), 119 (2006).

[3] О.П. Пчеляков, Ю.Б. Болховитянов, А.В. Двуреченский, Л.В. Соколов, А.И. Никифоров, А.И. Якимов, Б. Фойхтлендер. ФТП, 34 (11), 1281 (2000).

[4] J. Stangl, V. Holy, G. Bauer. Rev. Mod. Phys., 76, 725 (2004).

[5] D.J. Eaglesham, M. Cerullo. Phys. Rev. Lett., 64, 1943 (1990).

[6] Y.-W. Mo, D.E. Savage, B.S. Swartzentruber, M.G. Lagally. Phys. Rev. Lett., 65, 1020 (1990).

[7] A. Rastelli, H.V. Kanel, B.J. Spencer, J. Tersoff. Phys. Rev. B, 68, 115301 (2003).

[8] G. Medeiros-Ribeiro, A.M. Bratkovski, T.I. Kamins, D.A.A. Ohlberg, R.S. Williams. Science, 279, 353 (1998).

[9] F.M. Ross, R.M. Tromp, M.C. Reuter. Science, 286, 1931 (1999).

[10] M. Stoffel, A. Rastelli, J. Tersoff, T. Merdzhanova, O.G. Schmidt. Phys. Rev. B, 74, 155326 (2006).

[11] Z. Zhong, G. Bauer. Appl. Phys. Lett., 84, 1922 (2004).

[12] Z. Zhong, W. Schwinger, F. Schäffler, G. Bauer, G. Vastola, F. Montalenti, L. Miglio. Phys. Rev. Lett., 98, 176102 (2007).

[13] F. Hackl, M. Grydlik, M. Brehm, H. Groiss, F. Schäffler, T. Fromherz, G. Bauer. Nanotechnology, 22, 165302 (2011).
[14] F. Pezzoli, M. Stoffel, T. Merdzhanova, A. Rastelli, O.G. Schmidt. Nanoscale Res. Lett., 4, 1073 (2009).

[15] M. Grydlik, G. Langer, T. Fromherz, F. Schäffler, M. Brehm. Nanotechnology, 24, 105601 (2013).

[16] G. Vastola, M. Grydlik, M. Brehm, T. Fromherz, G. Bauer, F. Boioli, L. Miglio, F. Montalenti. Phys. Rev. B, 84, 155415 (2011).

[17] C. Dais, H. Solak, Y. Ekinci, E. Muller, H. Sigg, D. Grützmacher. Surf. Sci., 601, 2787 (2007).

[18] Z. Zhong, O. Schmidt, G. Bauer. Appl. Phys. Lett., 87, 133111 (2004).

[19] B. Yang, F. Liu, M. Lagally. Phys. Rev. Lett., 92, 025502 (2004).

[20] H. Hu, H. Gao, F. Liu. Phys. Rev. Lett., 109, 106103 (2012).

[21] J. Zhang, M. Stoffel, A. Rastelli, O. Schmidt, V. Jovanovic, L. Nanver, G. Bauer. Appl. Phys. Lett., 91, 173115 (2007).

[22] M. Salvalaglio, R. Backofen, A. Voigt, F. Montalenti. Nanoscale Res. Lett., 12 (1), 554 (2017).

[23] Zh. Smagina, P. Novikov, V. Zinovyev, N. Stepina, A. Dvurechenskii, V. Armbrister, V. Seleznev, P. Kuchinskaya. Phys. Status Solidi A, 210, 1522 (2013).

[24] P.L. Novikov, A.V. Nenashev, S.A. Rudin, A.S. Polyakov, A.V. Dvurechenskii. Nanotechnologies in Russia, 10, 192 (2015).

[25] P.N. Keating. Phys. Rev., 145, 637 (1966)

[26] S.A. Rudin, V.A. Zinovyev, A.V. Nenashev, A.Yu. Polyakov, Zh.V. Smagina, A.V. Dvurechenskii. Optoelectron. Instr. and Data Proc., 49, 461 (2013).

Редактор Г.А. Оганесян

\section{Nucleation of three-dimensional Ge nanoislands on pre-patterned $\mathrm{Si}(100)$ substrate}

S.A. Rudin ${ }^{1}$, Zh.V. Smagina ${ }^{1}$, V.A. Zinovyev ${ }^{1}$, P.L. Novikov $\mathbf{1 , 2}$, A.V. Nenashev $\mathbf{1 , 2}$, E.E. Rodyakina ${ }^{1,2}$, A.V. Dvurechenskii ${ }^{1,2}$

${ }^{1}$ Rzhanov Institute of Semiconductor Physics, Siberian Branch of Russian Academy of Sciences, 630090 Novosibirsk, Russia

${ }^{2}$ Novosibirsk State University, 630090 Novosibirsk, Russia

Abstract Abstract We study nucleation of three-dimensional Ge nanoislands formed on a pre-patterned $\mathrm{Si}$ substrate with an array of round pits. It is observed that $\mathrm{Ge}$ nanoislands prefer to nucleate inside the pits with sharp-shaped bottoms and on the outside the pits with flat-shaped bottoms. This effect is due to a different strain distribution over the Ge/Si interface of a pit bottom. The growth simulation shows that sharp-shaped pits have relaxed areas in the centers of the pit bottoms where nanoislands do precipitate. In contrast, flat-shaped pits have relaxed areas moved from the bottoms to the pit edges during the growth. 